\title{
Flying with a Safety Net: Use of REBOA to Enable Safe Transfer to a Level 1 Trauma Center
}

\author{
Robert Weir, Jeffery Lee, Shelly Almroth and Jodie Taylor \\ St. Anthony Summit Medical Center, Frisco, CO, USA
}

\begin{abstract}
Background: Using Resuscitative Endovascular Balloon Occlusion of the Aorta (REBOA) during air and ground transport requires coordination among the responding clinical team, transport team, and receiving surgical team. Here we describe the development of a REBOA transport program in a civilian medical system that demonstrates the value of REBOA as part of the toolkit for safe casualty transport.

Methods: The regional REBOA program was developed at St. Anthony Summit Medical Center (SASMC) in a multistep planning and training process to ensure coordination among the facilities and transport resources during trauma patient care. Retrospective record review was performed on all patients $(n=5)$ that received REBOA for transport from the Level 3 Trauma Center to the Level 1 Trauma Center, since the inception of the program in March 2019. Data were gathered from hospital electronic medical records.

Results: SASMC has transported five trauma patients under the REBOA program; all successfully arrived at the Level 1 Trauma Center to receive definitive care. The integrated arterial blood pressure monitoring capability in the REBOA catheter provided robust physiologic data to enable data-driven interventions during transport.

Conclusions: The REBOA program described here is a model of how REBOA can be used to enable safe transport between levels of care when, without REBOA, such transport might not be possible. The model is applicable during care of civilian trauma patients and combat casualties, where injured patients are initially treated in a prehospital or Role 1/2 environment but require transport to a Level 1 Trauma Center or Role 3+ for definitive care.
\end{abstract}

Keywords: REBOA; Non-compressible Hemorrhage; Patient Transport

\section{INTRODUCTION}

In the past 40 years, trauma systems in the United States have moved to a regionalized approach to health services including trauma care to provide optimal care through the rational distribution of medical services. Although difficult to isolate as a stand-alone factor, research has shown regional networks of trauma systems to be effective in reducing trauma related mortality [1]. Transfer of select patients from hospitals with fewer resources and capabilities to those with higher capabilities has numerous advantages; however, inherent in this approach is a period where the patient moves into a care environment with lower resources and capabilities, such

\section{Corresponding author:}

Jodie Taylor, 340 Peak One Drive, Frisco, CO 80443, USA.

Email: jodietaylor.md@gmail.com

(C) 2021 CC BY 4.0 - in cooperation with Depts. of Cardiothoracic/ Vascular Surgery, General Surgery and Anesthesia, Örebro University Hospital and Örebro University, Sweden as either a ground or air ambulance. In order to mitigate this risk, several approaches are routine, including preparing the patient for transfer, reducing exposure to the lower capability time using high-speed platforms such as helicopters, and the strategic addition of capabilities to transport platforms.

St. Anthony Summit County Regional Medical Center is a state-verified Level 3 Facility located in Frisco, Colorado, USA. As part of our ongoing process improvement efforts, we conducted an analysis of the capabilities at our facility, the affiliated Level 1 facility, and our transport platforms in an effort to identify opportunities for improved safety of trauma patients during transport. Transport times are managed through the use of a high-speed helicopter platform (Figure 1) that has both a higher speed and bypasses traffic delays, which can be significant due to a lack of redundant ground transportation corridors between the Level 3 in Summit County and the affiliate Level 1 in the Denver Metro Area (Figure 2). Transportation times in this system are generally around 1 hour; however, when weather conditions deteriorate, these times can be greatly extended as ground transportation 


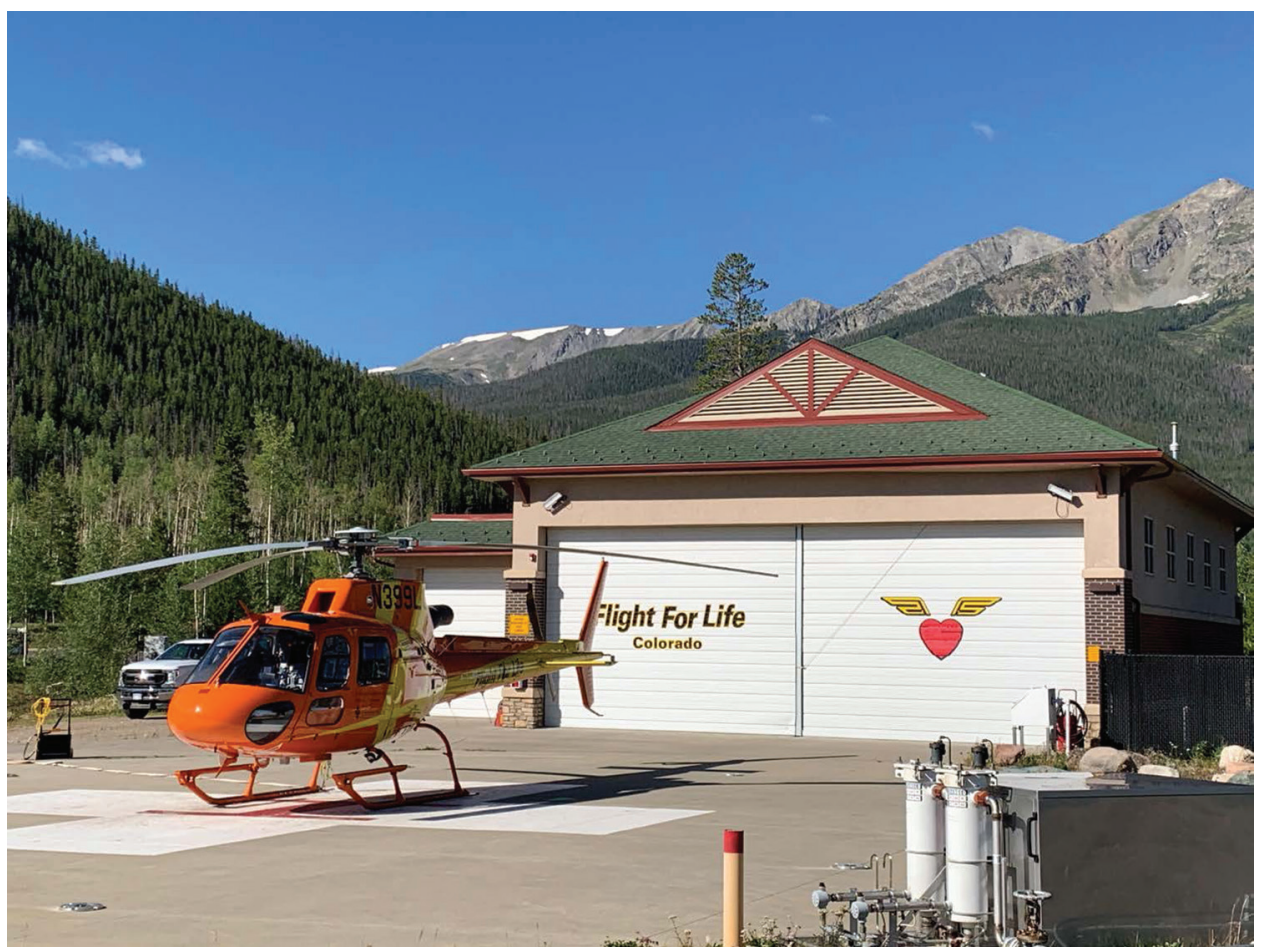

Figure 1 Flight for Life Colorado provides helicopter transport for critically ill patients from St. Anthony Summit Medical Center (Level 3 Trauma Center) to St. Anthony Hospital (Level 1 Trauma Center).

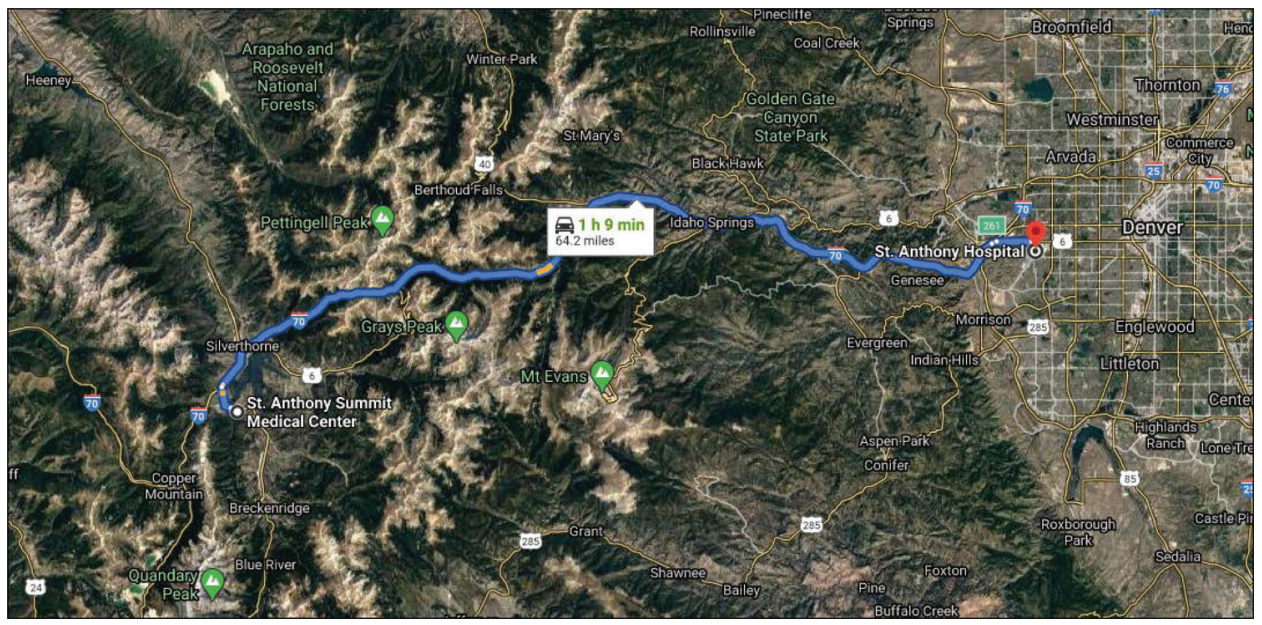

Figure 2 Ground route between St. Anthony Summit Medical Center (Level 3 Trauma Center) and St. Anthony Hospital (Level 1 Trauma Center). The typical ground transport time is around 1 hour with ideal weather and traffic. Helicopter flight time is significantly shorter. Poor weather can ground helicopters and increase drive time. Note the lack of redundant drive routes between hospital centers. Map image generated on Google Maps on 4 September 2020.

speed is greatly reduced by the same weather conditions which ground helicopters. There are numerous capability differences between our Level 3 and the Level 1 hospital; for trauma patients, the key factors that necessitate transfer are the need for definitive endovascular hemorrhage control, intensive care capabilities, and neurosurgical intervention. Access to these capabilities requires a facility transfer. We sought to leverage emerging technology to enable providers in transport platforms increased capability to intervene should patients decompensate during transport to the Level 1 center. As has been discussed by numerous authors, resuscitative endovascular balloon occlusion of the aorta (REBOA) has the potential to serve this function for bleeding trauma 


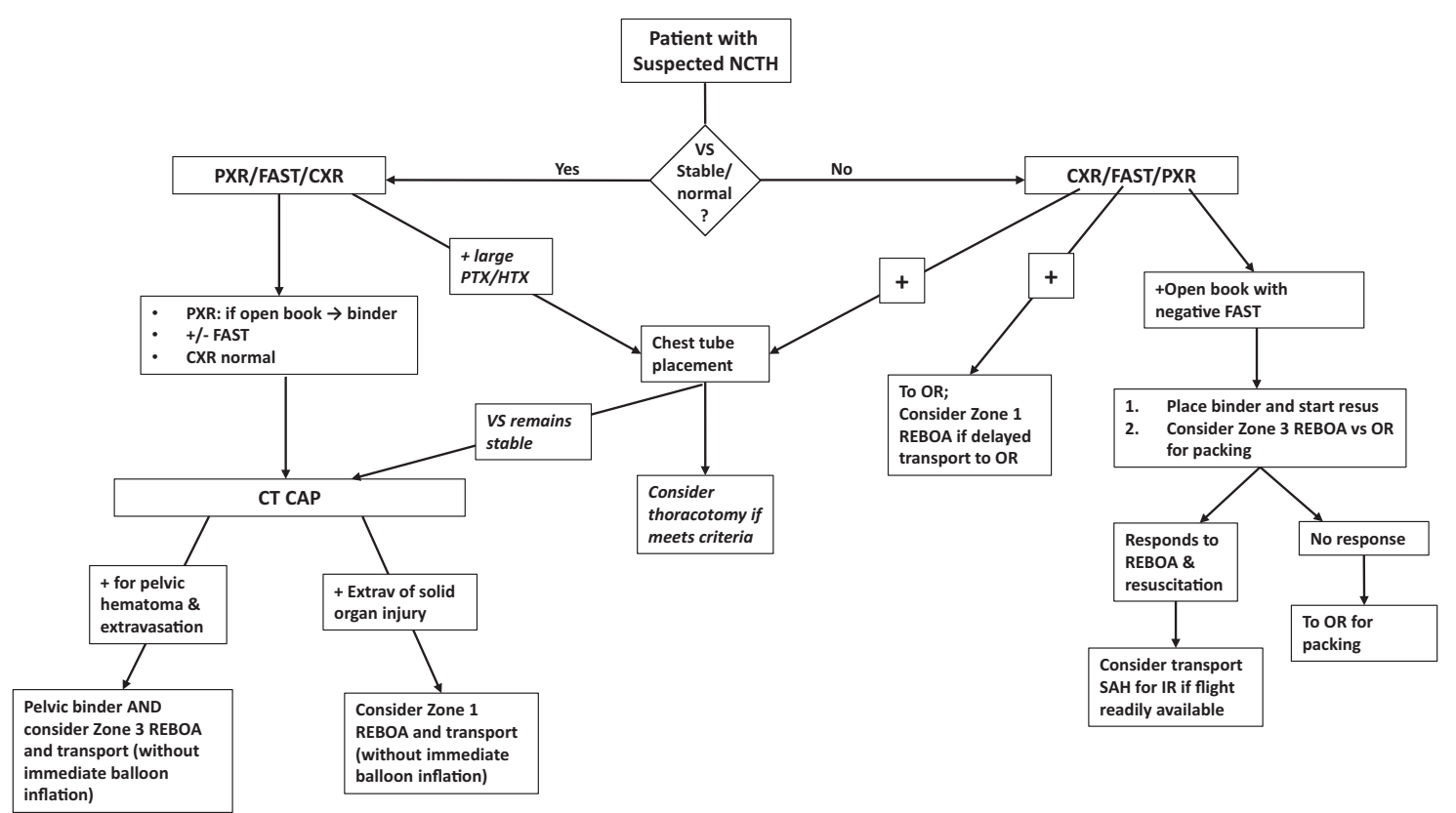

Figure 3 Course of care and transport decision protocol for the St. Anthony Summit Medical Center Regional REBOA Transport Program for patients with suspected non-compressible torso hemorrhage (NCTH). PXR, pelvic x-ray; FAST, focused assessment with sonography in trauma; CXR, chest x-ray; +, positive; VS, vital signs; PTX, pneumothorax; HTX, hemothorax; OR, operating room; CT CAP, computed tomography chest abdomen pelvis; REBOA, resuscitative endovascular occlusion of the aorta; SAH, St. Anthony's Hospital (Lakewood, CO, USA); IR, interventional radiology.

patients [2-4]. Use of endovascular balloon occlusion is rapidly evolving. Significant advances in devices have enabled its use in emergency care environments. Along with these device advances, clinical care approaches which leverage the full spectrum of REBOA-associated technology include: (a) timely invasive arterial monitoring, (b) established femoral artery access, (c) diagnostic imaging and surgical planning, (d) endovascular hemorrhage control, and (e) balloon occlusion to respond to decompensation [5]. This approach, termed "Step-Up REBOA", seeks to utilize the benefits of arterial access and endovascular interventions while avoiding the major limitation of prolonged use of aortic occlusion, distal ischemic injury. Here we report the development of a regional REBOA program developed to enable safe transfer of trauma patients from a Level 3 to a Level 1 Trauma Center and review the series of cases where we have implemented this protocol.

\section{METHODS}

\section{Development of the Regional REBOA Program}

New medical procedures are commonly first implemented in specialized centers and REBOA followed that pattern with initial clinical implementation at St. Anthony Hospital, an American College of Surgeons verified Level 1 Trauma Center, in early 2017. As REBOA rapidly moved into more diverse medical facilities, we embarked on a deliberate pathway to bring REBOA to the regional trauma system including the St. Anthony Summit Medical Center, a state-verified Level 3 Trauma Center, and the Flight for Life air ambulance, which transports patients from a regional base at St. Anthony Summit Medical Center to St. Anthony Hospital. In early 2018, after a year of experience with REBOA at the Level 1 Trauma Center, we initiated planning through a cross disciplinary approach involving personnel at both hospitals and the flight nurses responsible for patient care during transport. Trauma Surgery, Emergency Medicine, Vascular Surgery, Interventional Radiology, and the transportation medical director participated in the development of clinical practice guidelines and communication protocols. Prior to initiating the regional REBOA program, we conducted simulation-based training and validation in collaboration with the device manufacturer (Prytime Medical Devices, Inc., Boerne, TX, USA).

Our flight nurse crew was trained first, followed by the Emergency and Trauma Physicians. Simulation trainers for arterial access and REBOA manikins were used to confirm device performance and usability during flight, and the program was initiated in March 2019. We employed a multidisciplinary REBOA transport protocol at the Level 3 Trauma Center (Figure 3), protocols for the medical transport team, and integration with the existing REBOA protocol at the Level 1 Trauma Center.

Although our hospital has an air transport/critical care crew based at our center, the crew services a significant 
Table 1 Clinical data for each of 5 patients treated under the St. Anthony Summit Medical Center regional REBOA transport program.

\begin{tabular}{|c|c|c|c|c|c|}
\hline & Patient 1 & Patient 2 & Patient 3 & Patient 4 & Patient 5 \\
\hline Gender, age & M, 19 & $M, 51$ & M, 59 & $M, 68$ & $M, 21$ \\
\hline $\mathrm{MOI}$ & Snowboard fall & Mountain biking & $\begin{array}{l}\text { Motor vehicle } \\
\text { accident }\end{array}$ & Fall from height (8 feet) & Snowboard fall \\
\hline PPE & Helmet & Helmet & Seatbelt, rear pass & None & Helmet \\
\hline Scene/clinic BP/other & $\begin{array}{l}\text { 136/85, FAST } \\
\text { negative. }\end{array}$ & 117/73, P62 & & 146/90; P80 & $\begin{array}{l}\text { Walk-in - no } \\
\text { prehospital BP } \\
\text { available }\end{array}$ \\
\hline Scene GCS & 15 & 15 & 15 & 15 & 15 \\
\hline Pre-L3 time & $3 \mathrm{~h}$ & $30 \mathrm{~min}$ & $45 \min$ & $57 \mathrm{~min}$ & $8 \mathrm{~h}$ \\
\hline L3 Initial SBP, FAST & 116/75, FAST Pos. & 90/60; FAST positive & $\begin{array}{l}\text { 98/74; pulse 106; } \\
\text { FAST negative }\end{array}$ & $\begin{array}{l}\text { 119/81; P84; FAST } \\
\text { negative }\end{array}$ & 128/59; P63 \\
\hline Imaging & CT: pan scan & CXR, PXR, no CT & CXR, PXR, pan-scan & CT: pan-scan & CT: pan-scan \\
\hline L3 Blood & Yes & Yes & Yes & Whole blood & None \\
\hline Means of CFA access & Ultrasound guided & Ultrasound guided & Ultrasound guided & Ultrasound guided & Ultrasound guided \\
\hline TXA given? & No & Yes & Yes & Yes & No \\
\hline L3 diagnosis & $\begin{array}{l}\text { Grade } 5 \text { spleen, } \\
\text { grade } 2 \text { kidney (left) }\end{array}$ & $\begin{array}{l}\text { Mesenteric } \\
\text { hematoma with } \\
\text { active extravasation }\end{array}$ & $\begin{array}{l}\text { Retroperitoneal } \\
\text { bleed', sternal } \\
\text { fracture, rib fractures; } \\
\text { abdominal wall } \\
\text { dehiscence }\end{array}$ & $\begin{array}{l}\text { Right superior and } \\
\text { inferior pelvic rami } \\
\text { fracture; sacral fracture; } \\
\text { extraperitoneal } \\
\text { extravasation }\end{array}$ & Grade 4 spleen ${ }^{2}$ \\
\hline $\begin{array}{l}\text { L3 surgery avoided/ } \\
\text { deferred }\end{array}$ & Avoided & Deferred to L1 & Deferred to L1 & Avoided & Avoided \\
\hline $\begin{array}{l}\text { Zone of REBOA } \\
\text { insertion }\end{array}$ & Zone 1 & Zone 1 & Zone 1 & Zone 3 & Zone 1 \\
\hline Transport time & $32 \mathrm{~min} /$ helicopter & 24 min/helicopter & $32 \mathrm{~min} /$ helicopter & 70 min/ambulance & 65 min/ambulance \\
\hline Transport blood & None & Plasma & 2 units PRBCs & Plasma and PRBCs & None \\
\hline L1 blood & None & & MTP & No & None \\
\hline L1 diagnosis & $\begin{array}{l}\text { Grade } 5 \text { spleen, } \\
\text { grade } 2 \text { kidney (left) }\end{array}$ & $\begin{array}{l}\text { Mesenteric } \\
\text { hematoma with } \\
\text { active extravasation }\end{array}$ & $\begin{array}{l}\text { SB transection/ } \\
\text { avulsion mesentery }\end{array}$ & $\begin{array}{l}\text { R sup \& inf rami fracture; } \\
\text { sacral fracture; } \\
\text { extraperitoneal } \\
\text { hematoma }\end{array}$ & Grade 4 spleen injury; \\
\hline Hemorrhage diagnosis & $\begin{array}{l}\text { No active } \\
\text { extravasation on } C T\end{array}$ & $\begin{array}{l}\text { Small bowel } \\
\text { mesenteric } \\
\text { hemorrhage }\end{array}$ & $\begin{array}{l}\text { Retroperitoneal } \\
\text { hematoma, } \\
\text { mesenteric bleeding }\end{array}$ & $\begin{array}{l}\text { Extraperitoneal } \\
\text { hematoma }\end{array}$ & No extravasation on CT \\
\hline $\begin{array}{l}\text { Definitive surgical } \\
\text { procedure }\end{array}$ & $\begin{array}{l}\text { Splenic artery } \\
\text { embolization }\end{array}$ & $\begin{array}{l}\text { Ex lap, bowel } \\
\text { resection } \times 2\end{array}$ & $\begin{array}{l}\text { Exlap; bowel } \\
\text { resection; left in } \\
\text { discontinuity; } \\
\text { non-expanding } \\
\text { retroperitoneal } \\
\text { hematoma }\end{array}$ & Angiography & Angiography \\
\hline Nadir BP & & & & 55 & 120 \\
\hline REBOA complication & None & None & None & None & None \\
\hline Surgical complication & $\mathrm{N} / \mathrm{A}$ & None & Ml-death & $\mathrm{N} / \mathrm{A}$ & N/A \\
\hline Total AO time & None & None & None & None & None \\
\hline Disposition & $\begin{array}{l}\text { Discharge to home } \\
\text { day } 4\end{array}$ & $\begin{array}{l}\text { Discharge to home } \\
\text { day } 5\end{array}$ & Death & $\begin{array}{l}\text { Discharge to } \\
\text { rehab day } 4\end{array}$ & $\begin{array}{l}\text { Discharge to } \\
\text { home day } 4\end{array}$ \\
\hline
\end{tabular}

'Source of bleeding: non-surgical retroperitoneal muscular contusion. ${ }^{2}$ Hospital system protocol and Colorado state law dictates that all Grade 4 solid organ injuries are transferred to a L 1 Trauma Center for definitive care; in the case that Interventional Radiology is required, this resource is only available at the L 1 Trauma Centers. M, male; $M O l$, mechanism of injury; PPE, personal protective equipment; BP, blood pressure; FAST, focused assessment with sonography in trauma; GCS, Glasgow Coma Score; L3, Level 3 Trauma Center; CXR, chest X-ray; PXR, pelvic X-ray; CT, computed tomography; CFA, common femoral access; TXA, tranexamic acid; L 1, Level 1 Trauma Center; REBOA, resuscitative endovascular balloon occlusion of the aorta; PRBCs, packed red blood cells; MTP, massive transfusion protocol, SB, small bowel, Ex lap, exploratory laparotomy; $M I$, myocardial infarction; $A O$, aortic occlusion. 
portion of the central mountain region of Colorado and can be called away for other patient transporting needs. Patients did not enter the protocol if trained Flight for Life personnel were unavailable for transport, if blood pressure was sustained at $<90 \mathrm{mmHg}$, or if the patient required ongoing blood resuscitation to maintain systolic blood pressure (SBP) $>90 \mathrm{mmHg}$. In addition, hemodynamically normal and stable patients who could receive immediate helicopter load were not included in the REBOA protocol to ensure the fastest transport times.

While this protocol provides guidance for clinical decision-making, ultimately, the course of treatment and decision to place a REBOA catheter are at the discretion of the treating physician. The physician also has discretion to transfer patients as needed, although Colorado state law and regional transfer agreements dictate that certain injuries require transport to a Level 1 Trauma Center (e.g. Grade 4+ solid organ injury, spinal column injury with neurological deficit, epidural brain hemorrhage, etc.). During flight, the nurse flight crew manages the REBOA catheter by closely monitoring patient vital signs. Aortic occlusion is initiated when a patient's SBP drops below $90 \mathrm{mmHg}$. At the time of writing this manuscript, five transport cases have been performed under this program.

\section{Retrospective Record Review}

As part of this program, we established a process improvement initiative to review cases where REBOA is used during transport. Hospital electronic patient records $(n=5)$ from this process improvement initiative were analyzed for this case series.

\section{Ethical Approval and Informed Consent}

Authorization for the publication of this work was provided by the Catholic Health Initiatives Institute for Research and Innovation Institutional Review Board (CHIRB). Informed consent was not required for the publication of this article.

\section{RESULTS}

In the first 12 months after establishing our regional REBOA transport program, the intervention was performed in five patients (Table 1). All patients were male and the mechanism of injury was representative of the patients seen in this medical center, with alpine recreational mishaps and a motor vehicle collision resulting in blunt trauma with helmet and seat belt use appropriate to the activities. Time elapsed prior to admission to the Level 3 Trauma Center was widely variable. Concurrent with the establishment of the REBOA program, the Flight For Life critical care transport system also initiated blood product resuscitation capability with both fresh whole blood and component therapy. As a result the patients in this series received a combination of interventions for hypotension at the Level 3 Trauma Center including blood products (4/5), tranexamic acid (2/5), 7 Fr common femoral artery (CFA) access, and prophylactic placement of a REBOA Balloon Catheter (ER-REBOA $^{\mathrm{TM}}$, Prytime Medical Devices, Boerne, TX, USA). Blood products were also administered during transport in three of five patients. Initial care of these patients at the Level 3 Trauma Center was accomplished by a team consisting of surgeons and emergency medicine physicians who collaborated to initiate diagnostic and therapeutic procedures. All of the CFA access procedures were accomplished under ultrasound guidance, with the Emergency Medicine physicians responsible for performing this procedure.

All patients responded to resuscitative treatments at the Level 3 Trauma Center and during transportation resulting in no instances of inflation of the occlusion balloon. Imaging files from patients were available in the shared Emergency Medical Records system and available for physicians at both Level 3 and Level 1 Trauma Centers contemporaneously so that no repeat imaging was required once patients reached the Level 1 Trauma Centers. Upon arrival at the Level 1 Trauma Center, these patients underwent definitive treatment, which commonly was endovascular, to include additional diagnostic radiography and embolization. In total, two of the five patients did not have extravasation of contrast at the Level 1 center indicating that hemorrhage had stopped, and additional bleeding control was unwarranted. There were no complications associated with the use of REBOA or CFA access.

\section{DISCUSSION}

All of the patients in this case series had near normal scene Glasgow Coma Scale (GCS) scores and this, along with protective equipment use (primary injury prevention), contributed to the positive outcomes seen and the short course of hospitalization with 4 of 5 patients discharged within 5 days and most discharged home. Concurrently, with the development and implementation of the REBOA program, this regional trauma system also implemented a whole blood resuscitation program at the Level 3 Trauma Centre and in the helicopter platforms. This resulted in variable blood product use with balanced component therapy used in three patients, and whole blood used in one. These programs are complementary and best implemented in combination to leverage the strengths of each [6]. While none of the patients in this study progressed to the point where aortic occlusion was necessary, providing transport between the Level 3 and Level 1 Trauma Centers with an uninflated REBOA in place gives the transport team a tool to rapidly respond if a patient were to become hemodynamically unstable. We have experienced this situation twice in years past, before our REBOA Transport Program was 
in place; transport with REBOA provides additional safety to the patients and a higher level of confidence in the decision to transport the patients to the Level 1 Trauma Center with success. Furthermore, several benefits of this treatment were realized in addition to the added safety of having an intervention to treat emergent decompensation. These benefits included improved situational awareness from accurate, real-time arterial line pressure monitoring, and subsequent use of arterial access for endovascular interventions, and avoidance of morbid invasive procedures such as laparotomy and splenectomy [5]. As the practice of partial REBOA is more widely adopted, additional benefits can be realized. Partial REBOA gives the clinical team the ability to balance distal ischemia with proximal perfusion, thus extending the potential window for hemorrhage control [7]. The partial REBOA technique could be especially beneficial in transport cases that encounter extreme weather, traffic conditions, or long distances that increase the time needed to move a trauma patient from a Level 3 to Level 1 Trauma Center. Transport of the five patients resulted in three cases receiving definitive care through Interventional Radiology, a capability not available at the Level 3 Trauma Center. Of note, any hemodynamically unstable patients with a positive FAST are taken directly to the operating room without computed tomography (CT) scanning and do not enter the REBOA protocol. Patients are only transferred if they are hemodynamically stable and are determined by the sending physician to be best served by urgent interventional radiology or a more robust surgical intensive care unit.

CFA access is widely recognized as the most important step in enabling endovascular aortic occlusion and was a focus of our planning and training efforts $[5,8]$. While some trauma surgeons may not routinely perform ultrasound-guided vascular access, these procedures are common in the practice of Emergency Medicine physicians. To ensure access success, this REBOA program includes Emergency Medicine physicians who are responsible for gaining access, achieving a high success rate while also freeing the trauma surgeon to perform other tasks. Time from beginning to gain CFA access to having the REBOA catheter in place took less than 10 minutes in all cases, and typically occurs within 5-7 minutes. Since the placement of REBOA is ongoing simultaneously with additional patient preparation or treatment, there is low risk of the REBOA placement causing a delay in the departure of a patient for transfer. We found this to be the best practice and recommend against excluding Emergency Medicine physicians from REBOA procedures as this is counterproductive [9]. Early access gained by a provider proficient in ultrasoundguided access avoids the pitfalls of a difficult access caused by hypotension, low-flow, and vasospasm.

Non-compressible hemorrhage in the abdomen and pelvis remains a clinical challenge, one often best met at well-resourced Level 1 Trauma Centers. To facilitate safe transfer of patients to these capabilities, we instituted a transport REBOA program, and reviewed the indications, implementation and outcomes for patients treated using this protocol. We found this capability useful and safe as evidenced by patients arriving at the Level 1 Trauma Center, having been cared for in route according to the established plan. Initial care at the Level 3 center was significantly enhanced through our multidisciplinary approach. As noted by many, access has been shown to be the critical, often rate limiting, factor in the successful use of REBOA [10]. By using an interdisciplinary approach, this essential procedure is performed by those with experience, which in our center are the Emergency Medicine physicians who routinely practice vascular access under ultrasound guidance. We consider this inclusive approach to be a best practice rather than a surgeon-only approach to REBOA procedures, especially in settings like ours in which surgical personnel are limited or have not yet arrived at the hospital. While the ability to use REBOA if needed has value in bringing patients to the needed capability, this procedure is fundamentally linked to blood product resuscitation, and in our program these interventions have proved synergistic. While animal model studies often examine REBOA in the absence of blood product use, this does not reflect clinical care where transfusion and hemorrhage control are practiced together to achieve a common goal. Inclusion of state-of-the-art blood product transfusion practices is an essential part of the successful implementation of transport REBOA as it avoids the known limitations of each approach, thereby maximizing the impact of each.

In our Transport REBOA program, the occlusion of the aorta is the final step in a set of capabilities which we have found useful in the treatment of trauma patients. Initial vascular access enables us to transduce an accurate, real-time arterial pressure which is of use as resuscitation proceeds and additional diagnostic procedures such as CT imaging are conducted. The availability of an appropriate CFA access point ensures that diagnostic procedures can be conducted with the knowledge that a rescue procedure is rapidly available should the patient decompensate. Likewise, this access point has proven valuable at the Level 1 center where endovascular procedures to achieve definitive care are enabled. All of the patients in this case series had endovascular evaluation as part of their definitive care.

\section{CONCLUSION}

This regional approach to trauma care along with the use of helicopters to transport trauma patients are examples of trauma innovations where the transfusion of technologies between civilian and military trauma care have benefitted both [11]. The development and implementation of REBOA technology optimized for emergency and critical care environments is 
another example. The military, in collaboration with universities and industry, developed catheters designed for this environment, and their use has been refined as this technology and approach has been adopted in civilian centers [12]. We anticipate that, in addition to civilian regional trauma systems, military trauma systems may benefit from the successful development of protocols and training for use of REBOA during medical evacuation.

\section{Acknowledgements}

The authors thank Robin Cavill for clinical training on REBOA and support in setting up our REBOA program.

\section{Ethics Statement}

(1) All the authors mentioned in the manuscript have agreed to authorship, read and approved the manuscript, and given consent for submission and subsequent publication of the manuscript.

(2) The authors declare that they have read and abided by the JEVTM statement of ethical standards including rules of informed consent and ethical committee approval as stated in the article.

\section{Conflicts of Interest}

RW, JL, SA, and JT report no proprietary or commercial interest in any product mentioned or concept discussed in this article.

\section{Funding}

This research did not receive any specific grant from funding agencies in the public, commercial, or not-forprofit sectors.

\section{Author Contributions}

RW, JL, SA, and JT contributed to the development of the Regional REBOA Transport Program. JT performed patient record review and drafted the manuscript. RW, $\mathrm{JL}$, and SA critically edited the manuscript. All authors have read and approved the manuscript.

\section{Data Availability}

Hospital electronic patient records were analyzed for this case series. These data are not available for distribution.

\section{REFERENCES}

[1] Vali Y, Rashidian A, Jalili M, Omidvari AH, Jeddian A. Effectiveness of regionalization of trauma care services: a systematic review. Public Health. 2017;146:92-107.

[2] Sadek S, Lockey DJ, Lendrum RA, Perkins Z, Price J, Davies GE. Resuscitative endovascular balloon occlusion of the aorta (REBOA) in the pre-hospital setting: an additional resuscitation option for uncontrolled catastrophic haemorrhage. Resuscitation. 2016;107:135-8.

[3] Brown SR, Reed DH, Thomas P, Simpson C, Ritchie JD. Successful placement of REBOA in a rotary wing platform within a combat theater: novel indication for partial aortic occlusion. J Spec Oper Med. 2020;20:34-6.

[4] Reva VA, Horer TM, Makhnovskiy AI, Sokhranov MV, Samokhvalov IM, DuBose JJ. Field and en route resuscitative endovascular occlusion of the aorta: a feasible military reality? J Trauma Acute Care Surg. 2017; 83(1 Suppl 1):S170-S176.

[5] Vernamonti JP, Holcomb J, Mick NW, et al. 'Step Up' approach to the application of REBOA technology in a rural trauma system. Trauma Surg Acute Care Open. 2019;4:e000335.

[6] Butler FK Jr., Holcomb JB, Shackelford S, et al. Advanced resuscitative care in tactical combat casualty care: TCCC guidelines change 18-01:14 October 2018. J Spec Oper Med. 2018;18:37-55.

[7] Kemp MT, Wakam GK, Williams AM, et al. A novel partial resuscitative endovascular balloon aortic occlusion device that can be deployed in zone 1 for more than 2 hours with minimal provider titration. J Trauma Acute Care Surg. 2021;90:426-33.

[8] Matsumura Y, Matsumoto J, Kondo H, et al. Early arterial access for resuscitative endovascular balloon occlusion of the aorta is related to survival outcome in trauma. J Trauma Acute Care Surg. 2018;85:507-11.

[9] Brenner M, Bulger EM, Perina DG, et al. Joint statement from the American College of Surgeons Committee on Trauma (ACS COT) and the American College of Emergency Physicians (ACEP) regarding the clinical use of Resuscitative Endovascular Balloon Occlusion of the Aorta (REBOA). Trauma Surg Acute Care Open. 2018;3:e000154.

[10] Osborn LA, Brenner ML, Prater SJ, Moore LJ. Resuscitative endovascular balloon occlusion of the aorta: current evidence. Open Access Emerg Med. 2019;11:29-38.

[11] Committee on Military Trauma Care's Learning Health System and Its Translation to the Civilian Sector. A national trauma care system: integrating military and civilian trauma systems to achieve zero preventable deaths after injury. Washington: The National Academies Press; 2016.

[12] Rasmussen TE, Eliason JL. Military-civilian partnership in device innovation: development, commercialization and application of resuscitative endovascular balloon occlusion of the aorta. J Trauma Acute Care Surg. 2017;83:732-5. 